\title{
Keep your cool: burn calories
}

T he relevance of brown adipose tissue (brown fat) and its potential role in the prevention and management of obesity has been examined in a recent series of articles. That energy intake in excess of energy expenditure leads to the accumulation of adipose tissue is no surprise but, as noted in an editorial by Francesco Celi, “...evolutionary pressure has rewarded those [who are] able to store sufficient energy to survive famines ... the unrestricted availability of food represents an unnatural condition." (Celi, F. S. N. Engl. J. Med. 360, 553-556 [2009]). He suggests that this metabolic predisposition underlies the generally limited success of measures that aim to reduce energy intake (with the exception of bariatric surgery), and notes that current interventions to increase energy expenditure are also ineffective: exercise is difficult to sustain and pharmacologic treatments have shown disappointing results.

Re-enter brown adipose tissue, a fat depot present in rodents throughout life and identified in children, but long considered absent in adults. In contrast to white adipose tissue, which stores excess energy, brown fat contributes to basal and inducible energy expenditure. In newborn human babies, this tissue maintains core body temperature via nonshivering thermogenesis, in which expression of mitochondrial brown fat uncoupling protein 1 (UCP1) leads to uncoupled oxidative phosphorylation and decreased efficiency of cellular respiration. The inner membranes of mitochondria become leaky and release stored chemical energy as heat, instead of using it to generate ATP. Expression of UCP1 is upregulated within brown fat by the thyroid hormone tri-iodothyronine, $\beta_{3}$-adrenergic signaling, food intake and cold ambient temperatures. As little as $50 \mathrm{~g}$ of metabolically active brown fat could account for up to $20 \%$ of an adult's daily energy expenditure (Cypess, A. M. et al. N. Engl. J. Med. 360, 1509-1517 [2009]).

Brown adipose tissue was thought to involute during childhood, and its physiologic activity and physical presence only became recognized in adults when its appearance was noted as a 'nuisance' finding on ${ }^{18} \mathrm{~F}$-fluorodeoxyglucose (FDG) PET scans. Uptake of this tracer in cervical and subclavicular regions can represent residual areas of brown fat rather than neoplasia. The use of benzodiazepines, $\beta$-blockers, or warming of the room is required to reduce the signal from these areas and minimize false-positive findings.
The identification of brown adipose tissue in adults led Virtanen and colleagues to assess cold-induced glucose uptake in paracervical and subclavicular adipose tissue in five healthy individuals (Virtanen, K. A. et al. N. Engl. J. Med. 360, 1518-1525 [2009]). By exposing the volunteers to an ambient temperature of $17-19^{\circ} \mathrm{C}$ for $2 \mathrm{~h}$ before an ${ }^{18} \mathrm{~F}$-FDG PET-CT scan, the investigators were able to identify and obtain biopsy samples from areas that showed increased glucose uptake (by 15 -fold) in response to cold and thus demonstrate evidence that brown adipose tissue was present and metabolically active in healthy adult humans. The investigators estimated that if all of an individual's brown adipose tissue were continuously metabolically active it would burn the energy equivalent of $4.1 \mathrm{~kg}$ of (white) adipose tissue annually.

Cypess and colleagues then analyzed 3,640 consecutive ${ }^{18}$ F-FDG PET-CT images from 1,972 patients. The presence and amount of brown fat detected correlated with age, sex, and BMI. Although no cold-exposure protocol was used in this study, the investigators did document the outdoor temperature at the time of the scans. They found metabolically active brown adipose tissue in $7.5 \%$ of women and $3.1 \%$ of men. The probability of detecting brown fat inversely correlated with age, outdoor temperature, and BMI.

In a third manuscript, van Marken Lichtenbelt and colleagues studied 24 healthy men using ${ }^{18} \mathrm{~F}-\mathrm{FDG}$ PET-CT imaging under neutral ambient temperatures and during mild cold exposure (van Marken Lichtenbelt, W. D. et al. N. Engl. J. Med. 360, 1500-1508 [2009]). They identified brown fat in 23 of 24 individuals and confirmed that its metabolic activity was significantly lower in overweight than in lean individuals, and that resting metabolic rate was positively correlated with glucose uptake in brown fat.

We now know that brown fat is more prevalent in lean adults than obese ones, and is involved in energy metabolism. While the ability to upregulate brown fat activity by lowering ambient temperatures may have some potential for increasing energy expenditure, the mere recognition that this tissue exists and is metabolically active in adult humans provides a long-lost, and still underappreciated, target for controlling the worldwide obesity epidemic.

doi:10.1038/nrgastro.2009.88

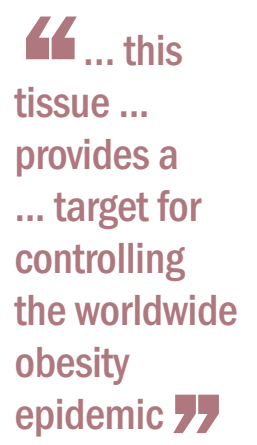

Stephen B. Hanauer is the Editor-in-Chief of Nature Reviews Gastroenterology \& Hepatology.

Competing interests The author declared no competing interests. 